\title{
Innovative Approach in the Use of Traditional Aangi of Haryana
}

\author{
Dr. Parul Gill ${ }^{1} \mid$ Dr. Saroj S. Jeet Singh ${ }^{2}$ \\ ${ }^{1}$ Department of Textile and Apparel Designing, CCS Haryana Agricultural University, Hisar, Haryana, India \\ 2 Department of Textile and Apparel Designing, CCS Haryana Agricultural University, Hisar,Haryana, India
}

To Cite this Article

Dr. Parul Gill and Dr. Saroj S. Jeet Singh, "Innovative Approach in the Use of Traditional Aangi of Haryana", International Journal for Modern Trends in Science and Technology, 6(9S): 126-131, 2020.

Article Info

Received on 25-August-2020, Revised on 08-September-2020, Accepted on 12-September-2020, Published on 18-September-2020.

\section{ABSTRACT}

Traditional choliof Haryana, locally known as aangi, is rare to be seen in today's time. This upper garment was worn by women before the adoption of kurta. It was tied at back with two pairs of strings and worn by teaming with daaman. It was usually stitched by using ten to twelve pieces of fabric. Aangis were usually multicolored as these were made from various leftover fabric pieces available at home. That is why they sometimes lacked any symmetry of colour. These different pieces were joined together by finishing the seams with magji, rick-rack, gotta or embroidery to give it a unified look. A lot oftime was spent in preparation and decoration of this attire. Aangi was a highly intricate garment depicting the talent of women in this craft. The present study was conducted to assess the market potential of this traditional choli of Haryana in thismodern era.For this purposeanaangiblouse was constructed by the researcher in which constructional features of the traditional aangiwere incorporated. Cotton blend fabrics of yellow, red and blue colours were used as base material while herring bone stitch and mirrors were used for embellishmentas per experts' suggestions. The aangiblousewas assessed for marketability by 200 respondents including Indian as well as foreign visitors and stall owners/exporters in the International Trade Fair, Delhi. Acceptability of aangiblouse was found to be excellent on all parameters i.e., traditional value (WMS 3.89), aesthetic appeal (WMS 3.75), workmanship (WMS 3.69), colour combination (WMS 3.67), utility (WMS 3.64) uniqueness (WMS 3.57) and fabric (WMS 3.41). On an average acceptability was found to be excellent. This aangiblouse can be teamed with a transparent saree for creating a unique style statement. Some respondents suggested to modify aangi as top or to use original form of aangi under transparent top.

KEYWORDS: Traditional, Choli,Aangi, Haryana,Aangiblouse

\section{INTRODUCTION}

Indiacurrently has 28 states and each statehasits own unique culture.Costumes of India vary from region to region depending on different ethnicity, geography, climate, and cultural influences.Different styles of clothes worn by people in different regions are known to be their traditional dressing. They sew, embroider or decorate them in special designs to make them look distinct, charming and unique in their own way, representing the places they belong to. (1).

India's rich heritage of ornamentation of fabrics dates back to the epic ages. The splendour and versatility of Indian hand printed, hand painted and hand-embroidered fabrics glitter in every part of the world even today(2). 
Haryana is one small state. Haryanvi people are known for their simple living. The life of Haryanvi women have always been hard and busy. They have always been too busy taking care of their home, children, animals, and fields as well. Despite a busy schedule they used to find time for hand work like embroidery. Although it has not been given due recognition but Haryanvi women were very artistic and creative. Their artistic tastes and skills are reflected in theirtraditional garments. The resources available to them were limited which heightened their creativity. They had mastered the art of creating garments using every single left over piece at their homes. The perfect example of such garments is 'aangi' - the traditional choli of Haryana. Aangiis rare to be seen in today's time. This upper garment was worn by women before the adoption of kurta. This garment was a true example of creating best out of waste.

But with industrialization and the invasion of western culture, the clothing styles of people have changed due to which aangi is not worn by modern Haryanvi women. Such a wonderful piece of traditional art work is now diminishing. It deserves to be preserved. In order to revive this dying art, the present study was conducted with the following objectives:

1. To explore and modify the traditional aangifor contemporary use for marketing.

2. To assess the market potential of modifiedaangiblouse.

\section{METHODOLOGY}

\section{Participants}

Thirty rural women above 50 years of age, 30 Clothing and Textile experts and 200 visitors at International Trade Fair, Pragati Maidan, New Delhi were the participants in the study at different stages.

\section{Tools}

Interview Schedule: The information was gathered using two interview schedules at different stages of the study. The interview schedules were used to gather following specific information:

Interview schedule 1- Preliminary information from rural women about traditional aangi with reference to fabric, colour, embellishment techniques and cultural importance.
Interview schedule 2- Information related to the preferences and suggestions of experts regarding colour-combinations, fabrics and embellishment for modification.

Questionnaire and Visitor's Book: These two tools were developed with the help of a business management expert for assessing market potential of modified aangi blouse. The seven product features were aesthetic appeal, utility, workmanship, uniqueness, color- combination, fabric and traditional value. The product features were assessed using four quantum scale.

\section{RatingWeightage}

Excellent

Very Good

Good

Poor

\section{Statistical Analysis}

The articles were finally rated on the basis of their WMS as per the following scale-

\section{WMSRating}

3.26-4.00

2.56-3.25

$1.76-2.50$

$1.00-1.75$

Procedure

\section{Excellent}

Very Good

Good

Poor
Elderly rural women of Haryana were contacted personally using snowball technique. The purpose of the study was explained to them. Preliminary information about this traditional garment with reference to fabric, colour, embellishment techniques and cultural importance was gathered by interviewing these women.Traditional aangis were collected from villages of Haryana for reference.Expert views were obtained regarding modifying aangi into possible contemporary clothing use. Suggestions of experts regarding colour combination, fabric and embellishment for modification in aangi were also recorded. The final aangiblouse was prepared on the basis of experts' suggestions. The article with the suggested modifications was prepared by the researcher herself. Substantial time and efforts were devoted in learning the process of making the aangiby close observation of the original specimens. Finally, the 
traditional aangi was reinvented as anaangi blouse ofmodern look to enhance its suitability for modern market.

The prepared aangi blouse was displayed in the International Trade Fair, Delhi. The aangi blouse was got assessed by Indian and foreign visitors as well as exporters and stall owners. The assessment was done for acceptability in terms of aesthetic appeal, utility, workmanship, uniqueness, color combination, fabric and traditional value in domestic as well as foreign market.

\section{RESULTS AND DISCUSSION}

\section{Aangi (Backless choli)}

It was a backless blouse or choli locally known as aangi tied at back with two pairs of strings and worn by teaming with daaman. It had usually ten to twelve pieces of fabric. Breast cup was made by joining two to three on grain pieces. The breast pieces were either three square pieces or one square and one rectangular piece joined with hands to convert into a single piece. For heavy busted gathers or pleats were also used at the lower part of the cup. Invariably a very deep ' $\mathrm{V}$ ' neckline visible in aangi was finished using two straight bands joined in the centre. It is important to mention that no special cutting was done for sleeves to make armhole but a straight piece was joined with a gasket (under arm piece) to give it sufficient ease. Sometimes two extra triangular shoulder pieces were also used. Aangis were usually multicolored, made by using all fabric pieces available at home, even sometimes without any symmetry/combination. Though different pieces were joined together but were made to look part of a single garment by finishing the seams with magji, rick-rack, gotta or embroidery. After stitching all the pieces together in a garment form the inner side was sometimes finished with lining using hemming or running stitch. There were two pair of dori (strings) for tying at back of neck and at waist. These strings hold the garment in shape which could be tightened as per requirement. Dori could be of thick cotton yarn or of fabric strip made into cord or piping. Dori ends were decorated with fabric pieces (funde). A hanging rectangular vertical piece attached at centre, covered the naval button. Naval piece's edges were turned and finished with blanket stitch. Gotth (piping) was used on neckline, sleeve hems and waist. It is clearly indicated that aangi was a highly intricate garment depicting the talent of women in this craft and women used to spend lots of time in preparation and decorating this attire.

\section{Decoration of Aangi:}

a) Applique work- Applique work was generally done on breast pieces and sleeves, using white fabric on dark background. Motifs used were 6-8 petal flowers and rows of leaves.

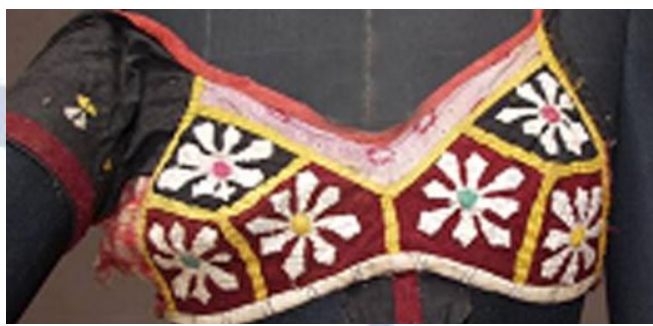

(a)

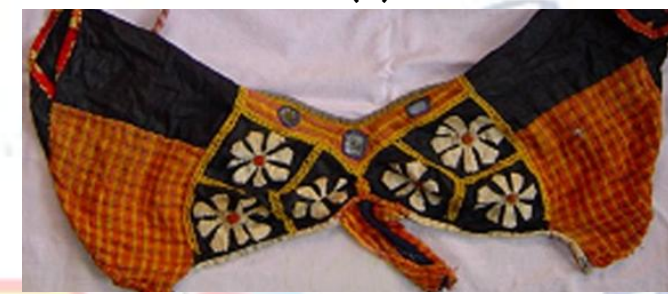

(b)

Figure 1(a) and (b) Decoration of Aangi with appliqué work

b) Mirror work - Mirrors were applied generally at neckline, sleeves and at the centre of the floral appliqué.

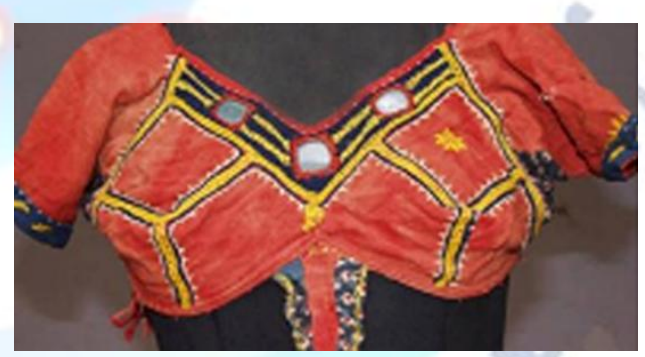

\section{Figure 2 Decoration of Aangi with} appliqué work

c) Gotta work - Silver colour gotta was applied at neckline and breast, either straight or in zigzag form which was known as bankra. Pemak was generally applied at sleeves. Gotta flowers were also seen on each breast.

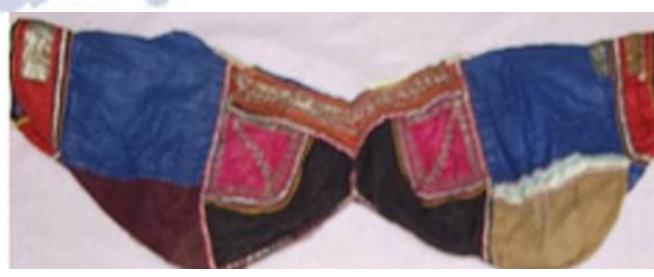

(a) 


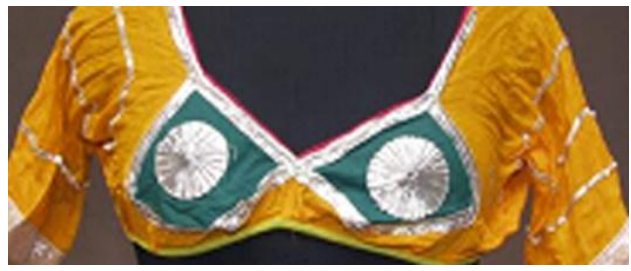

(b)

Figure 3 (a) and (b) Decoration of Aangi withgotta work

d) Embroidery - Herring bone and feather stitches were worked on neckline and breast pieces with six strands of yellow colour cotton thread to hide seamlines. Some really fine work aangis were almost filled with phulkari using long darning stitch, herringbone, two parallel rows (simultaneously made) of pseudo back stitch, chain stitch and triangle stitch.

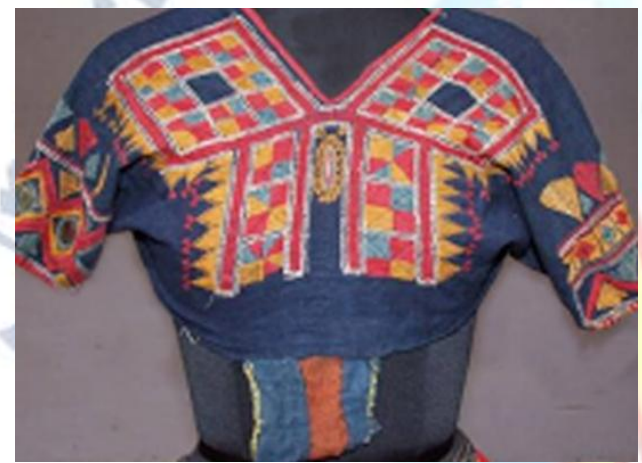

Figure 4Decoration of Aangi with embroidery

e) Magji - Magji of various colours was used to decorate the aangi. Simultaneously 2, 3 or 5 colours were used e.g. coca-cola, maroon, white, blue and yellow or red, white and blue. Magzi work was done at breast pieces, neck and sleeves.

Traditionally reja of blue and maroon colour was commonly used but white check and striped reja was also seen. After arrival of mill fabric, printed fabric and plain voile fabric was also used. The aangis made up of voile fabric were very vibrant with yellow, red and orange colours. But these were generally used only for tying Teel (dresses) to be sent in dowry and not for covering upper body (as kurtas came into existence).

\section{Modifying Traditional Aangi}

Table 1 shows suggestions of the expertsregarding thepossible contemporary applications of aangi.Using aangi as saree blouse was suggested by maximum number of experts (72.73\%). It was suggested that aangi in its original form could be used as blouse with a transparent/chiffon saree. While few others suggested to modify aangi as top
$(36.36 \%)$ or to use original form of aangi under transparent top $(22.73 \%)$. Table 1Possible contemporary applications of
aangi $(\mathrm{N}=22)$

\begin{tabular}{|l|c|c|}
\hline \multicolumn{1}{|c|}{$\begin{array}{c}\text { Contemporary } \\
\text { applications }\end{array}$} & F & \% \\
\hline Saree blouse & 16 & $\mathbf{7 2 . 7 3}$ \\
\hline $\begin{array}{l}\text { Blouse under transparent } \\
\text { top }\end{array}$ & 5 & 22.73 \\
\hline Top & 8 & 36.36 \\
\hline
\end{tabular}

Multiple responses

It is apparent from Table 2 that cotton blend fabric $(83.33 \%)$ of medium thickness $(83.33 \%)$ was recommended for aangi which is available for blouses in market, which should not be transparent to serve the purpose (modesty). Most of the respondents recommended traditional bright colours $(73.33 \% 860.0 \%$ respectively) to give more traditional look to blouse.

Table 2: Preferences of expertsregarding fabric and colour for aangi $(\mathrm{N}=30)$

\begin{tabular}{|l|c|c|}
\hline Fabric & Frequency & Percentage \\
\hline Fabric thickness \\
\hline Thick & 5 & 16.66 \\
\hline Med & 25 & $\mathbf{8 3 . 3 3}$ \\
\hline Thin & -- & -- \\
\hline Fabric type & \multicolumn{3}{|l|}{} \\
\hline Cotton & 5 & 16.66 \\
\hline Blend & 25 & $\mathbf{8 3 . 3 3}$ \\
\hline Synthetic & -- & -- \\
\hline Fabric Colour & \\
\hline Traditional & 22 & $\mathbf{7 3 . 3 3}$ \\
\hline Any colour & 8 & 26.66 \\
\hline Bright & 18 & 60.0 \\
\hline Dull & -- & -- \\
\hline
\end{tabular}

Multiple responses

It is evident from table 3 that embroidery was suggested by most of experts (66.66\%) followed by mirror work $(56.66 \%)$, applique work $(33.33 \%)$, beads (13.33\%) and sequins (16.66\%). Beads and gotta work was also suggested by $13.33 \%$ experts. 
Table 3 Preferences of experts regarding embellishment for aangi $(\mathrm{N}=30)$

\begin{tabular}{|l|c|c|}
\hline Embellishment & F & \% \\
\hline Embroidery & $\mathbf{2 0}$ & $\mathbf{6 6 . 6 6}$ \\
\hline Mirrors & 17 & 56.66 \\
\hline Beads & 4 & 13.33 \\
\hline Sequins & 5 & 16.66 \\
\hline Lace & -- & -- \\
\hline Appliqué & 10 & 33.33 \\
\hline Rings & & \\
\hline Gotta & 4 & 13.33 \\
\hline Stones & 1 & 3.33 \\
\hline
\end{tabular}

Multiple responses

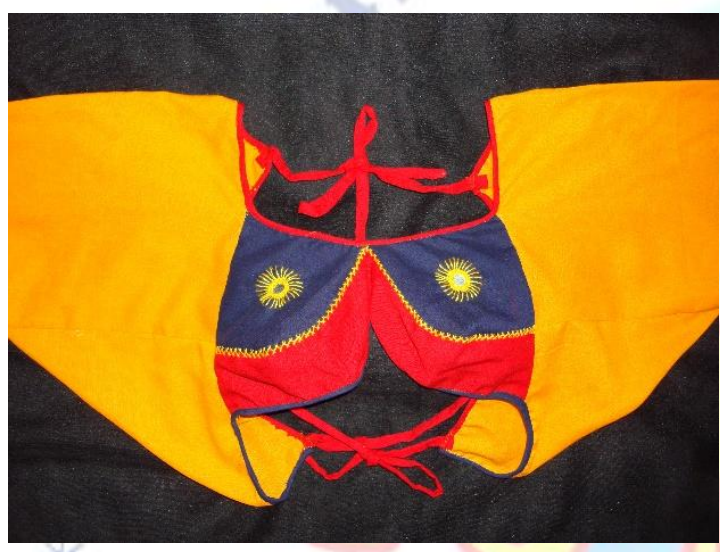

Figure 5 Aangi blouse

Figure 5 shows the new aangi blouse. The aangi blouse was prepared using constructional features of the traditional aangi. Cotton blend fabrics of yellow, red and blue colours were used as base material while herring bone stitch and mirrors were used for embellishment. This aangi can be teamed with a transparent saree for creating a unique style statement.

General information of the respondents contacted for final assessment of the products for social and market acceptability is shown in Table 4. The table makes it clear that male respondents $(55 \%)$ were slightly higher in number as some females $(45 \%)$ were hesitant in responding. Maximum number $(50.5 \%)$ of respondents were from Delhi, followed by Haryana (20\%), Punjab $(20 \%)$ and abroad (16\%). Remaining 11.5 per cent included visitors from, Nagaland, U.P, Rajasthan, Bombay and Chandigarh as well as the Foreigners and an NRI.
Table 4 General information of the respondents ( $N=\mathbf{2 0 0})$

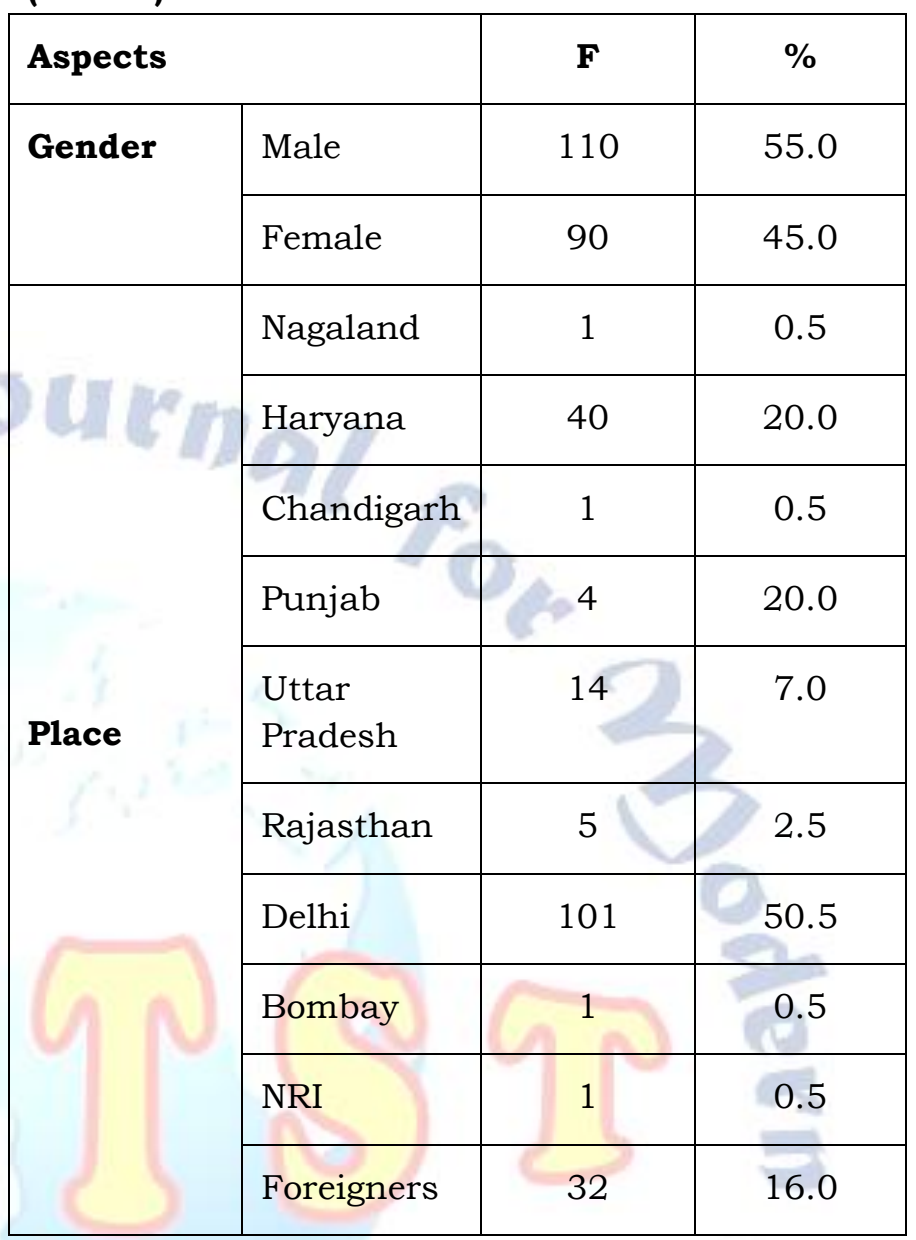

As is evident from the data in table 5 acceptability of aangi was adjudged excellent on all parameters i.e., traditional value (WMS 3.89), aesthetic appeal (WMS 3.75), workmanship (WMS 3.69), colour combination (WMS 3.67), utility (WMS 3.64) uniqueness (WMS 3.57) and fabric (WMS 3.41). On an average acceptability was found to be excellent with WMS 3.66.

Table 5 Acceptability level of Aangi

\begin{tabular}{|l|c|c|c|c|}
\hline Features & $\begin{array}{c}\text { Exce } \\
\text { llent }\end{array}$ & $\begin{array}{c}\text { V. } \\
\text { Good }\end{array}$ & Good & WMS \\
\hline $\begin{array}{l}\text { Aesthetic } \\
\text { appeal }\end{array}$ & 151 & 49 & -- & 3.75 \\
\hline Utility & 143 & 43 & 14 & 3.64 \\
\hline Workmanship & 138 & 62 & -- & 3.69 \\
\hline Uniqueness & 122 & 70 & 8 & 3.57 \\
\hline
\end{tabular}




\begin{tabular}{|l|c|c|c|c|}
\hline $\begin{array}{l}\text { Colour } \\
\text { combination }\end{array}$ & 137 & 59 & 4 & 3.67 \\
\hline Fabric & 92 & 98 & 10 & 3.41 \\
\hline $\begin{array}{l}\text { Traditional } \\
\text { value }\end{array}$ & 179 & 21 & -- & 3.89 \\
\hline
\end{tabular}

WMS-Weighted Mean Score

1-1.75 (Poor), 1.76-2.50 (Good), 2.51-3.25 (V. good), 3.26-4.00 (Excellent)

\section{CONCLUSION}

It can be concluded from the present study that traditional aangi of Haryana has the potential of revival if appropriate and sufficient efforts are made. The article if produced in modified form to suit the taste and needs of contemporary wearers, will be accepted readily by the modern market. Aangi blouse is proven to be accepted. Entrepreneurs in the field of handicrafts and garments can reinvent aangi in various new designs with traditional touch to earn profits.

\section{REFERENCES}

[1]https://www.indiaonline.in/about/culture/cos tumes-of-india

[2] https://www.epch.com

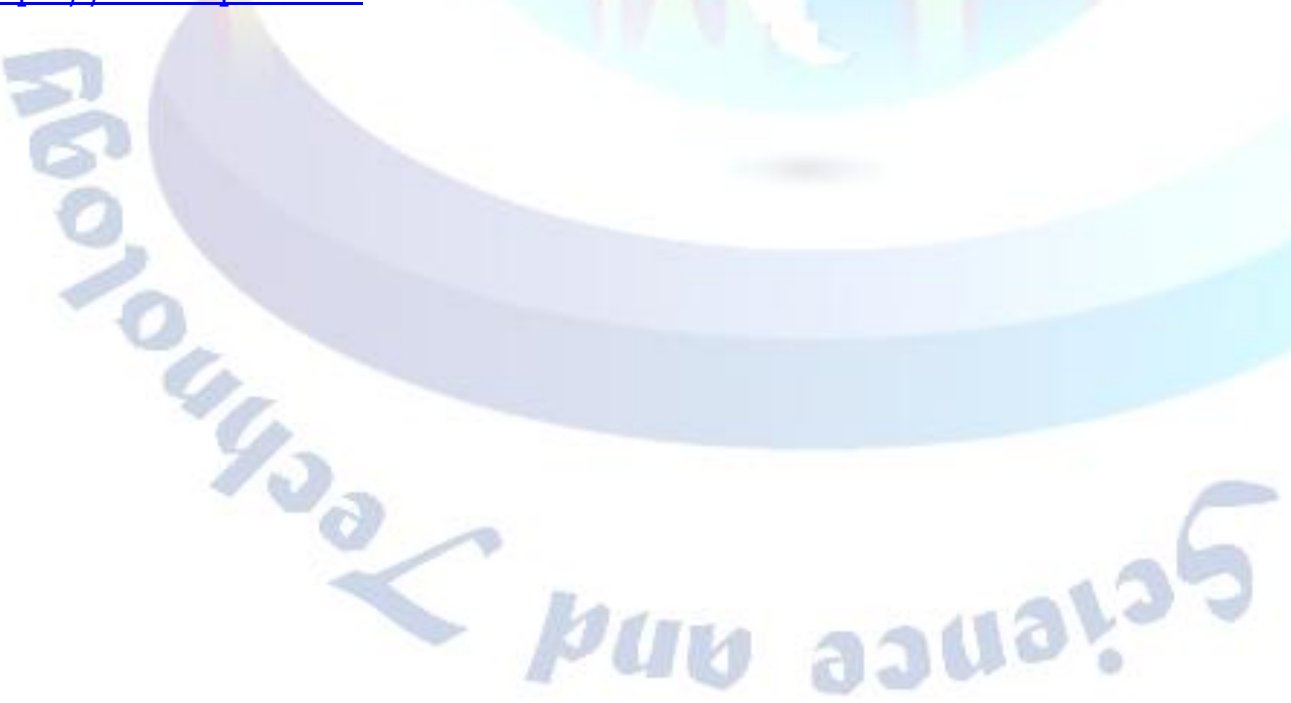

